COPYRIGHT @ 2018 INTERNATIONAL JOURNAL OF SCIENCE DENTISTRY | AVAILABLE ONLINE http://www.periodicos.uff.br/index

\title{
TOXINA BOTULÍNICA EM ADULTOS COM BRUXISMO PRIMÁRIO: UMA DISCUSSÃO CRÍTICA DA LITERATURA
}

\section{BOTULINUM TOXIN IN ADULTS WITH PRIMARY BRUXISM:}

\section{A CRITICAL DISCUSSION OF LITERATURE}

Luiza Abreu Sendra, Especialista em Prótese Dentária e Implantodontia pela Odontoclínica Central do Exército

Caroline Montez Lima dos Santos, Aluna de Mestrado em Clínica Odontológicada Universidade Federal Fluminense

Eliane dos Santos Porto Barboza, Mestre e Doutora em Periodontia, Professora associada da Faculdade de Odontologia da Universidade Federal Fluminense

Categoria do trabalho: Artigo de revisão

Palavras-chave: toxina botulínica; bruxismo

Keywords: botulinumtoxin; bruxismo

Autor correspondente: Eliane Porto Barboza

Endereço para correspondência:Faculdade de Odontologia - UFF - Rua Mário Santos Braga, 28 - Centro - Niterói - RJ

e-mail:elianeporto.uff@gmail.com 
COPYRIGHT @ 2018 INTERNATIONAL JOURNAL OF SCIENCE DENTISTRY | AVAILABLE ONLINE http://www.periodicos.uff.br/index

\section{INTRODUÇÃO}

O bruxismo é definido por repetitiva atividade muscular caracterizada por ranger ou apertar os dentes e/ou por travamento ou movimento da mandíbuladurante o sono, bruxismo do sono (BS),ou durante a vigília, bruxismo da vigília (BV)(LOBBEZOO F et al,, em 2013). Diferentemente do bruxismo secundário, o bruxismo primário não tem qualquer associação comdesordem médica ou medicamentosa.O diagnóstico do bruxismo pode ser realizado pelo relato do paciente, exame clínico e, no BS, por polissonografia (GUAITA M e HOGL B, 2016). A etiologia do bruxismo é complexa e controversa e possui associação com múltiplos fatores de risco. A maioria dos autores afirma que se trata de uma doença de origem central, dando menor importância aos fatores periféricos (MANFREDINI et al., 2011). Seus efeitos negativos vão desde fadiga, dor edesgastes dentários a perdas de dentes e implantes, dores de cabeça, lesões ósseas e disfunções temporomandibulares em casos severos (DEMJAHA Get al., 2019). Na ausência de tratamento para a causa do bruxismo, essa parafunção é gerida focando na prevenção do progresso do desgaste dentário e na redução dos seus sinais e sintomas. Aconselhamentos, estratégias de hábitos, placas oclusais, medicações e estimulação elétrica mostraram resultados heterogêneos na sua resolução(GUAITA M e HOGL B, 2016).As toxinas botulínicas são exotoxinas da bactéria Clostridium botulinum que inibem a liberação de neurotransmissores, reduzindo a contração muscular. Essas neurotoxinas injetáveistêm sido usadas clinicamente para diversas indicações na área de saúde. No entanto, a utilização no aparelho mastigatório ainda não consta na bulae seu uso tem sido "off label"(BRIN et al., 2014). O desenvolvimento de diretrizes de tratamento requer protocolos e estudos avaliando os efeitos de diferentes modalidades terapêuticas. $\mathrm{O}$ presente trabalho tem como objetivo discutir de forma crítica a literatura existente sobre o uso de toxina botulínica (TB) na gestão do bruxismo primário em adultos.

\section{DISCUSSÃO DA LITERATURA}

Pioneiramente, em 1990, ZANDIJCKEe MARCHAUreportaram o uso da TBtipo A (TB/A) para o controle de um caso de bruxismo secundário. Cada músculo temporal e masseter recebeu $25 \mathrm{U}$ e, após 5 dias, houve uma melhora que durou 8 
COPYRIGHT @ 2018 INTERNATIONAL JOURNAL OF SCIENCE DENTISTRY | AVAILABLE ONLINE http://www.periodicos.uff.br/index

semanas. Depois de 2 semanas, a paciente recebeu novamente um total de $100 \mathrm{U}$ e obteve melhora similar por 12 semanas.

Revisando a literatura, até a presente data,talvez pelo custo, pela forma de aplicação e pelo efeito temporário do medicamento, ainda há poucos estudos clínicos sobre o uso da TB em adultos com bruxismo primário.Não há trabalho científico exclusivamente sobre bruxismo em vigília. A maioria dos trabalhos apresenta amostras pequenas e relacionadas ao bruxismo do sono.Nenhuma publicação comparou os efeitos da diluição e a consequente dispersão da toxina no músculo alvo. Todos os autores obtiveram resultados positivos com a TB e apontaram a necessidade de mais estudos controlados randomizados. Porém, os trabalhos apresentam grande variação metodológica, usando diferentes toxinas, aplicando em distintas localizações, administrando doses variadas, avaliando emintervalos de tempos diversose utilizando diferentes formas de avaliação.

\section{Diferentes Toxinas}

As toxinas botulínicas são medicamentos de natureza biológica que apresentam diferentes processos de manufatura, formulações e métodos para determinar suas unidades de atividade. Isso resulta em um conjunto específico de interações entre cada tipo de toxina e o tecido injetado. Portanto, cada tipo de TB precisa ser individualmente investigada para estabelecer sua eficácia e seus parâmetros de segurança em cada indicação de uso (BRIN et al., 2014). Um ensaio feito com o americano Botox (OnabotulinumtoxinA) pode não apresentar os mesmos resultados que a toxina britânica Dysport (AbobotulinumtoxinA), a alemã Xeomin (IncobotulinumtoxinA),a italiana Vistabex (OnabotulinumA) ea coreana Neurotox (OnabotulinumA). Não há na literatura uma comparação clínica entre essas toxinas.

\section{Pontos e Músculos da Mastigação}

A literatura sobre manejo do bruxismo mostra uma ausência de protocolo na seleção de pontos e músculos para aplicaçãoda TB. A maioria dos trabalhos usa 3 pontos de aplicação nos masseteres (BOLAYIR et al., 2005;LEE et al., 2010; REDAELLI, 2011; ZHANG et al., 2016 e AL-WAYLI, 2017).ASUTAY, 2017 aplicou em 4 pontos dos masseteres. Outros ensaios foram feitos com aplicação nos masseteres e temporais: 3 pontos nos masseteres e 2pontos nostemporais (GUARDA-NARDINI et 
COPYRIGHT @ 2018 INTERNATIONAL JOURNAL OF SCIENCE DENTISTRY | AVAILABLE ONLINE http://www.periodicos.uff.br/index

al., 2008), 2pontos nos masseteres e 3 pontos nostemporais(ONDO, 2018)e 1 ponto em cada masseter e3 pontos nos temporais(JADHAO et al., 2017).

Um estudo comparou os resultados da aplicação da toxina Neurotroxsó nos masseteres com os resultados da aplicação nos masseteres e temporais, em 20 pacientes com diagnóstico clínico de BS. Cada músculo recebeu 25U de toxina distribuídas em 3 pontos. Em avaliações por videopolissonografias, antes e após 4 semanasda intervenção, os autores não constataram redução na frequência ou no ritmo da atividade muscular. No entanto, houve redução no pico da amplitude da eletromiografia, na intensidade dos episódios. Nos questionários, 9 pacientes reportaram redução do rangido e 18 relataram diminuição da rigidez mandibular matinal. Com esses resultados, os autores concluíram que a TB/A isolada no masseter é efetiva no controle do BS em pelo menos 1 mês, reduzindo a intensidade das contrações dos músculos do fechamento mandibular.Estudos como este precisam ser ampliados, pois podem garantir o uso da TB com um número mínimo de injeções no manejo do bruxismo (SHIM et al., 2014).

Injeções guiadas por ultrassomforam comparadas com as realizadasapósmarcação clínica em 3 pontos do masseterde 20 pacientes bruxistas. Como ultrassom, os autores constataram que65\% dos indivíduos apresentavam variações anatômicas da glândula salivar. Houve erro da localização clínica do ponto mais anterior do masseter em $40 \%$ dos casos e, em $20 \%$ dos pacientes, a agulha de insulina de $8 \mathrm{~mm}$ de comprimento não alcançou o músculo, sendo necessário utilizar agulha de $13 \mathrm{~mm}$. Após 3 meses da aplicação de TB/A não especificada, apenas $20 \%$ dos pacientes relatou ainda ter BS. Os autores concluíram que o ultrassom pode ser uma boa ferramenta para guiar as injeções de toxina botulínica nos masseteres, ajudando a evitar complicações(QUEZADA-GAON et al., 2016).No entanto, os trabalhos que realizaram a aplicação da TB/A em pontos selecionados por estimativa clínica, através de palpação e medições, obtiveram melhora da sintomatologia do bruxismo com muito poucos ou nenhum registro de efeitos indesejáveis (BOLAYIR et al., 2005; GUARDANARDINI et al., 2008; LEE et al., 2010; REDAELLI, 2011; SHIM et al., 2014; ZHANG et al., 2016; ASUTAY, 2017; JADHAO et al., 2017; AL-WAYLI, 2017; ONDO, 2018).A maior parte dos estudos não esclarece o tamanho da agulha utilizada (GUARDA-NARDINI et al., 2008; LEE et al., 2010; ZHANG et al., 2016; ASUTAY, 2017; JADHAO et al., 2017; AL-WAYLI, 2017). BOLAYIR et al. (2005) mencionaram 
COPYRIGHT @ 2018 INTERNATIONAL JOURNAL OF SCIENCE DENTISTRY | AVAILABLE ONLINE http://www.periodicos.uff.br/index

ter usado agulha de $0.8 \mathrm{~mm}$ comprimento, o que parece um engano do autor. REDAELLI(2011) relatou a utilização de agulha de 8mm. SHIM et al.(2014) e ONDO(2018) informaram ter usado agulhas de 0.5 polegada, que corresponde aproximadamente a $13 \mathrm{~mm}$. Nenhum trabalho mencionou a profundidade de introdução da agulha nas injeções.

\section{Doses}

A dose recomendada de TB para uso odontológico é deaté $100 \mathrm{U}$ por sessão (SRIVASTAVA et al., 2015). Os trabalhos existentes utilizaram doses por paciente bem variadas: 14U (REDAELLI, 2011); 20U (AL-WAYLI, 2017 e ASUTAY, 2017); 25U (ZHANG et al., 2016 ) em cada masseter; 25U em cada músculo(SHIM et al., 2014); $80 \mathrm{U}$ nos masseteres (LEE et al., 2010);50U em cada masseter (BOLAYIR et al., 2005); $30 \mathrm{U}$ em cada masseter e $20 \mathrm{U}$ em cada temporal somando $100 \mathrm{U}$ por paciente (GUARDA-NARDINI et al., 2008 e JADHAO et al., 2017) e 60U em cada masseter e 40U em cada temporal, somando200U por paciente (ONDO, 2018). Todos estes estudos mostraram a dose usada e o seu efeito, sem correlacionar a dose com a extensão ou a duração da paresia.

REDAELLI (2011) adequouas doses da TB/AVistabexde acordo com a satisfação do paciente em relação à melhora da sintomatologia do bruxismo. Inicialmente, aplicou $2 \mathrm{U}+2 \mathrm{U}+4 \mathrm{U}$ em 3 pontos de cada masseter de 20 pacientes e, após 15 dias, através de questionário, apenas 2 consideraram bom o resultado. $\mathrm{O}$ autor acrescentou mais $2 \mathrm{U}$ em cada um dos 6 pontos de aplicação desses pacientes, aplicando um total de $14 \mathrm{U}$ para cada masseter. Após 15 dias, todos relataram boa ou muito boa melhora dos sintomas. Com esse resultado inicial, o autor aplicou 14U divididas em 3 pontos de $4 \mathrm{U}, 4 \mathrm{U}$ e $6 \mathrm{U}$ em masseteres de 100 pacientes. Na avaliação depois de 2 semanas, apenas 5 pacientes não tiveram resultado satisfatório e receberam mais $2 \mathrm{U}$ em cada ponto e, depois do mesmo período, apenas 1 obteve melhora. Efeitos colaterais significantes não foram constatados. Após 4-6 meses, dos 120 pacientes, 42 não foram reavaliados e, dos 78 que retornaram, 68 solicitaram novas aplicações no masseter. Com esses resultados, Redaelli sugeriu como melhor dose efetiva sem efeitos colaterais $14 \mathrm{U}$ para cada masseter e, para casos de músculos mais fortes, $20 \mathrm{U}$. 
COPYRIGHT @ 2018 INTERNATIONAL JOURNAL OF SCIENCE DENTISTRY | AVAILABLE ONLINE http://www.periodicos.uff.br/index

\section{Intervalos de Tempo}

Alguns estudos avaliaram os resultados das injeções de TB apenas uma vez após 15 dias(REDAELLI, 2011), 1 mês (SHIM et al., 2014) ou 6 meses(GUARDANARDINI et al, 2008). A maioria dos trabalhos realizou mais de uma avaliação após intervenção, porém seguiram intervalos diferentes um do outro(BOLAYIR et al., 2005; LEE et al., 2010; ZHANG et al., 2016; ASUTAY, 2017; JADHAO et al., 2017; ALWAYLI, 2017; ONDO, 2018). Os intervalos entre as avaliações e o tempo total de acompanhamento interferem nos resultados, pois a toxina tem um efeito variável ao longo do tempo. Segundo ASUTAY et al.(2017),em média, os pacientes começaram a sentir os efeitos da TB em 12.24 +- 2.02 dias e a perdê-los $4.76+-1.01$ meses.

O trabalho com maior tempo de acompanhamento comparou a evolução do tratamento de 25 pacientes submetidos a 20UI de TX/A Botox em cada masseter (grupo I) com 25 pacientes submetidos ao tratamento convencional sem injeções (grupo II). Todas os participantes foram avaliados com questionário usando Escala Analógica Visual (VAS) antes e após 3 semanas, 2, 6 e 12 meses. A média de dor inicial foi de 7.1+-0.72 para o grupo I e de 7.5+-0.66 para o grupo II. Após 6 e 12 meses, os pacientes tiveram uma média de dor 0.2+-0.51 no grupo I e 2.1+-0.74 no grupo II. Os resultados mostraram que houve melhora maior no grupo que recebeu TB e que a melhora obtida após 6 meses foi mantida até 1 ano após o tratamento. No entanto, o autor relatouque o grupo submetido somente ao tratamento convencional não mostrou melhora da dor com o tempo, o que não está de acordo com os números apresentados. SegundoAl-Wayli, sem mostrar medição do número de eventos de bruxismo, os resultados sugeriram que as injeções de toxina reduziram a pontuação média de dor e o número de eventos do bruxismo, diminuindo a atividade muscular. (AL-WAYLI, 2017)

\section{Avaliação dos Efeitos}

A maioria dos trabalhos realizou questionários como método de avaliação dos efeitos da TB (BOLAYIR et al., 2005; GUARDA-NARDINI et al., 2008; LEE et al., 2010; REDAELLI, 2011; SHIM et al., 2014; ASUTAY, 2017; JADHAO et al., 2017; AL-WAYLI, 2017; ONDO, 2018). O questionário é uma forma subjetiva para avaliação e dificulta a obtenção de conclusões seguras. Poucos estudos usaram, além de questionários, métodos de avaliação objetivos como polissonografia(SHIM et al., 2014), 
COPYRIGHT @ 2018 INTERNATIONAL JOURNAL OF SCIENCE DENTISTRY | AVAILABLE ONLINE http://www.periodicos.uff.br/index

eletromiografia(LEE et al, 2010), análise digital da oclusão (JADHAO et al., 2017) e medida clínica da abertura máxima (ASUTAY, 2017).

O estudo de ZHANG et al.(2016) foi realizado sem questionários. Os autores utilizaram uma ferramenta digital de análise oclusal, o 'I-motionOcclusal Force Analyser', para avaliar a eficácia terapêutica e o efeito na força oclusal da aplicação de 25U de toxina botulínica não especificada em 3 pontos de masseteres de pacientes com DTM e bruxismo. Randomicamente, 10 pacientes receberam a toxina, 10 receberam solução salina e 10 não receberam injeções. A força máxima oclusal do grupo com TB teve valor mínimo após 3 meses e, após 6 meses, ficou mais baixo que o valor antes do tratamento. A duração da oclusão diminuiu significativamente no grupo com TB após 3 meses. Assim como no trabalho de JADHAO et al.(2017), a força oclusal diminuiu nos 3 grupos. JADHAO verificou melhora mais significativa no grupo com TB e ZHANG et al., nos grupos com TB e com placebo, o que, segundo os autores, demonstrou a importância psicológica na melhora dospacientes. ZHANG et al. concluíram que a TB oferece vantagens no tratamento da DTM, reduzindo a força oclusal, mas lembraram a importância da intervenção psicológica no sucesso dos tratamentos.

LEE et al. (2010)avaliaram o efeito de injeções de TB/A em 12 pacientes que relataram ter BS. Randomicamente, 6 indivíduos receberam 80U de TB Dysport em 3 pontos de cada masseter e 6 bruxistas receberam $0,8 \mathrm{ml}$ de solução salina da mesma forma. Antes das injeções e 4, 8 e 12 semanas após, os pesquisadores aplicaram questionários e realizaram gravações da eletromiografia do masseter e do temporal. Apesar do trabalho relatar que os sintomas do bruxismo foram avaliados por questionário, as perguntas realizadas se tratavam da frequência do episódios de bruxismo e os dois grupos relataram redução do número de episódios. Segundo os autores, os sintomas de bruxismo reduziram nos dois grupos e os eventos de bruxismo no masseter diminuíram significativamente no grupo injetado com TB. Em todas as avaliações, entre os dois grupos não houve diferença no temporal. Os autores concluíram que os resultados obtidos sugeriram que injeções com TB reduziram o número de eventos do bruxismo, provavelmente mediado pelo seu efeito na redução da atividade muscular e não no sistema nervoso central. Adicionalmente, LEE et al. apontaramque o procedimento com injeções de TB foram efetivos no BS. Trabalhos como este, que avaliam objetivamente os músculos masseter e temporal, podem 
COPYRIGHT @ 2018 INTERNATIONAL JOURNAL OF SCIENCE DENTISTRY | AVAILABLE ONLINE http://www.periodicos.uff.br/index

descartar a possibilidade de compensação domúsculo temporal ao eleger somente o masseter para aplicação da toxina.

\section{Efeitos Indesejáveis}

ASUTAY (2017) aplicou 20U de Botox em 25bruxistas e relatou efeitos adversos em 2 pacientes. No entanto, o autor não esclareceu quais foram tais efeitos.ONDO (2018) aplicou 200U de Botox em 13 pacientes e constatou a alteração no sorriso de 2 pacientes.Em geral, as reações adversas das injeções de TB são incomuns e localizadas. Os efeitos colaterais mais comuns das injeções orofaciais da TB são alterações na consistência salivar e inadvertida fraqueza dos músculos da face, da deglutição e da fala. São complicações localizadas e dose dependentes (SRIVASTAVA et al., 2015).

Recentes estudos em animais mostraram uma preocupação com o risco de osteopenia do côndilo e do osso alveolar associado ao uso de toxina botulínica em músculos da mastigação.No entanto, a diferença dos padrões ósseos de humanos e animais limita a extrapolação de resultados. RAPHAEL et al.(2014)publicaram um estudo em que2 radiologistas compararam o trabeculado dos côndilos de 7 mulheres submetidas a 2 sessões de TB/A com o de 9 mulheres que nunca receberam essa alternativa de tratamento. Em tomografias cone-beam, os avaliadores notaram uma diminuição da densidade óssea apenas nas pacientes expostas à TB/A. Os autores questionaram a significância dos achados e concluíram que estudos com maioramostra e acompanhamento mais longo ainda são necessários. É preciso investigar por quanto tempo esse possível efeito persiste, se representa algum risco e se existe uma dose de toxina que não o provoque.

Uma revisão sistemática para explorar as propriedades musculares apósinjeções de TB/A foi conduzida por MATHEVON et al.(2015). Em humanos,os autores constataram que, 1 ano após a intervenção, a recuperação muscular permanecia incompleta. Havia redução da espessura muscular em ultrassom 2D e atrofia muscular em análise volumétrica de ressonância magnética.AL-WAYLI, em 2017, também constatou permanência de efeitos da TB após 1 ano de acompanhamento. Se o efeito obtido de enfraquecimento muscular é o desejado, a sua permanência não parece ser um problema. 
COPYRIGHT @ 2018 INTERNATIONAL JOURNAL OF SCIENCE DENTISTRY | AVAILABLE ONLINE http://www.periodicos.uff.br/index

Nenhum trabalho clínico de uso da TB em músculos da mastigação explorou a indesejável formação de anticorpos antitoxina botulínica que diminuem a duração e o efeito terapêutico da toxina. Mais estudos para esclarecer os mecanismosda TB paraelaboração de estratégias de controle dos seus efeitos indesejáveis ainda são necessários.

\section{CONCLUSÕES}

$\mathrm{Na}$ ausência de melhora com tratamentos convencionais e mais conservadores, a TB, após o esclarecimento e consentimento do paciente que não possua contraindicação, parece uma alternativa eficaz para a gestão do bruxismo.

Há lacunas importantes no entendimento do manejo do bruxismo com o uso da toxina botulínica. Mais estudos sobre o assunto são ainda necessários.

\section{REFERÊNCIAS BIBLIOGRÁFICAS}

Lobbezoo F, Ahlberg J, et al.Bruxism defined and graded: an international consensus.J Oral Rehabil. 2013 Jan;40(1):2-4.

Guaita M, Högl B. Current Treatments of Bruxism. Curr Treat Options Neurol. 2016 Feb;18(2):10.

Manfredini D, Bucci MB, et al.Bruxism: Overview of current Knowledge and Suggestions for dental implants planning. The jornal of craniomandibular practice. 2011; 29(4): 1-9.

Demjaha G, Kapusevska B, et al.Bruxism Unconscious Oral Habit in Everyday Life. Open Access Maced J Med Sci. 2019 Mar 14;7(5):876-881.

Brin MF, James C, et al.Botulinum toxin type A products are not interchangeable: a review of the evidence.Biologics. 2014 Oct 6;8:227-41

Van Zandijcke M, Marchau MM. Treatment of bruxism with botulinum toxin injections.J NeurolNeurosurg Psychiatry. 1990 Jun;53(6):530.

G. Bolayir, E. Bolayir, et al.Botulinum toxin type-A practice in bruxism cases. Neurology Psychiatry and Brain Research. 2005 Jan;12(1):43-45.

Lee SJ, McCall WD Jr, et al.Effect of botulinum toxin injection on nocturnal bruxism: a randomized controlled trial.Am J Phys Med Rehabil. 2010 Jan;89(1):16-23. 
Redaelli A. Botulinum Toxin A in bruxers. One year experience. Saudi Med J. 2011 Feb;32(2):156-8.

Zhang LD, Liu Q, et al.Occlusal force characteristics of masseteric muscles after intramuscular injection of botulinum toxin A(BTX - A)for treatment of temporomandibular disorder.Br J Oral Maxillofac Surg. 2016 Sep;54(7):736-40.

Al-Wayli $\mathrm{H}$. Treatment of chronic pain associated with nocturnal bruxism with botulinum toxin. A prospective and randomized clinical study. J ClinExp Dent. 2017 Jan 1;9(1):e112-e117.

Asutay F, Atalay Y, et al.The Evaluation of the Clinical Effects of Botulinum Toxin on Nocturnal Bruxism, Pain Res Manag. 2017;2017:6264146.

Guarda-Nardini L, Manfredini D, Salamone M, Salmaso L, Tonello S, Ferronato G. Efficacy of botulinum toxin in treating myofascial pain in bruxers: a controlled placebo pilot study.Cranio. 2008 Apr;26(2):126-35.

Ondo WG, Simmons JH, et al.Onabotulinum toxin-A injections for sleep bruxism: A double-blind, placebo-controlled study.Neurology. 2018 Feb 13;90(7):e559-e564.

Jadhao VA, Lokhande N, et al.Efficacy of botulinum toxin in treating myofascial pain and occlusal force characteristics of masticatory muscles in bruxism. Indian $\mathrm{J}$ Dent Res. 2017 Sep-Oct;28(5):493-497.

Shim YJ, Lee MK, et al.Effects of botulinum toxin on jaw motor events during sleep in sleep bruxism patients: a polysomnographic evaluation.J Clin Sleep Med. 2014 Mar 15;10(3):291-8.

Quezada-Gaon N, Wortsman $\mathrm{X}$, et al.Comparison of clinical marking and ultrasound-guided injection of Botulinum type A toxin into the masseter muscles for treating bruxism and its cosmetic effects.J CosmetDermatol. 2016 Sep;15(3):23844.

Srivastava S, Kharbanda S, et al.Applications of botulinum toxin in dentistry: A comprehensive review. Natl J Maxillofac Surg. 2015 Jul-Dec;6(2):152-9.

Raphael KG, Tadinada A, et al.Osteopenic consequences of botulinum toxin injections in the masticatory muscles: a pilot study.J Oral Rehabil. 2014 Aug;41(8):555-63.

Mathevon L, Michel F, et al.Muscle structure and stifness assessment after botulinum toxin type A injection. A systematicreview. Ann PhysRehabil Med. 2015 Dec;58(6):343-50. 